\title{
A Normal-Mode Approach to Jovian Atmospheric Dynamics*
}

\author{
RICHARD K. ACHTERBERG AND ANDREW P. INGERSOLL \\ Division of Geological and Planetary Sciences, California Institute of Technology, Pasadena, California
}

(Manuscript received 3 November 1988, in final form 1 March 1989)

\begin{abstract}
We propose a nonlinear, quasi-geostrophic, baroclinic model of Jovian atmospheric dynamics, in which vertical variations of velocity are represented by a truncated sum over a complete set of orthogonal functions obtained by a separation of variables of the linearized quasi-geostrophic potential vorticity equation. A set of equations for the time variation of the mode amplitudes in the nonlinear case is then derived. We show that for a planet with a neutrally stable, fluid interior instead of a solid lower boundary, the barotropic mode represents motions in the interior, and is not affected by the baroclinic modes. One consequence of this is that a normalmode model with one baroclinic mode is dynamically equivalent to a one layer model with solid lower topography. We also show that for motions in Jupiter's cloudy lower troposphere, the stratosphere behaves nearly as a rigid lid, so that the normal-mode model is applicable to Jupiter. We test the accuracy of the normal-mode model for Jupiter using two simple problems: forced, vertically propagating Rossby waves, using two and three baroclinic modes, and baroclinic instability, using two baroclinic modes. We find that the normal-mode model provides qualitatively correct results, even with only a very limited number of vertical degrees of freedom.
\end{abstract}

\section{Introduction}

Numerical models of Jupiter's zonal jets and large circulating ovals generally focus on horizontal structure. The vertical structure of wind and pressure, and interactions with Jupiter's fluid interior, are either ignored or handled with simplifying assumptions. For example, the 1-layer models (Williams 1975; Maxworthy and Redekopp 1976; Williams and Yamagata 1984; Williams and Wilson 1988) have only a single degree of freedom in the vertical. The $1 \frac{1 / 2}{2}$ layer models (Ingersoll and Cuong 1981; Marcus 1988; Dowling and Ingersoll 1988,1989 ) have only one degree of freedom associated with a thin upper weather layer. Latitudinally varying bottom topography simulates the steady zonal motions in a much deeper, adiabatic lower layer, but only the top layer motions are free to change. Other models (Williams 1979; Read and Hide 1984; Read 1986) have two or more vertical degrees of freedom, but assume a flat, rigid lower boundary. The upper boundary condition-expressing the fact that the density approaches zero at the top of the atmosphere-is not adequately treated in any of the models. Not only are the models quantitatively inaccurate, but they fail qualitatively to simulate many important processes

\footnotetext{
* Contribution number 4721 from the Division of Geological and Planetary Sciences, California Institute of Technology.

Corresponding author address: Richard K. Achterberg, Division of Geological and Planetary Sciences, California Institute of Technology, Pasaderia, CA 91125.
}

such as baroclinic instability and vertical propagation of energy. For instance, Gierasch et al. (1979) and Conrath et al. (1981) have shown that the presence or absence of the deep lower layer has a large effect on the growth rates of baroclinic instabilities, indicating that a baroclinic model of Jupiter needs the proper lower boundary condition.

The sources of the deficiencies are both observational and conceptual. The visible and infrared observations of the Jovian planets (summarized by Ingersoll et al. 1984) provide detailed information down to cloud top levels $(500$ to $700 \mathrm{mb}$ ). Winds are defined by tracking clouds in Voyager images (Ingersoll et al. 1981; Mitchell et al. 1981; Hatzes et al. 1981; Limaye et al. 1982; Limaye 1986; Mac Low and Ingersoll 1986; Dowling and Ingersoll 1988). Infrared observations measure the abundances of water, ammonia and other gases for pressures less than about 5 bars (Conrath and Gierasch 1986; Bjoraker et al. 1986a,b), and provide information about the thermal structure above the cloud tops (Gierasch et al. 1986). The Voyager radio occultation experiment probed the region from $1 \mathrm{mb}$ to $1000 \mathrm{mb}$ (Lindal et al. 1981), providing profiles of temperature versus pressure, but no direct information about winds. Below the ammonia clouds one must rely on wet and dry adiabatic extrapolations, using cosmochemical and spectroscopic estimates for the amount of water and other condensables (Pollack et al. 1986; Bjoraker et al. $1986 \mathrm{a}, \mathrm{b})$. The net effect of these uncertainties has been to discourage investigators from examining the conceptual problems associated with the upper and lower boundary conditions. We wish to rectify this situation. 
Our goal is to develop a framework for systematically introducing multiple degrees of freedom in the vertical into models of large-scale Jovian atmospheric dynamics. The effect of observational uncertainties can be explored systematically. In this paper, we treat only the simplest dynamical problems, using them as a test of our model. In a later paper we will apply the framework to a nonlinear time-dependent numerical model of the Great Red Spot and its interactions with the zonal jets.

We propose a normal-mode (Galerkin) approach to the three-dimensional (baroclinic) quasi-geostrophic (QG) equations, assuming that the stably-stratified section of atmosphere being modeled lies on top of an infinitely deep, adiabatic fluid, which is allowed to have a zonal flow. This deep fluid provides a simple description for the effects of Jupiter's interior on the atmosphere. Vertical variations of the horizontal streamfunction are represented by a summation over orthogonal eigenfunctions resulting from a separation of variables on the linearized $Q G$ potential vorticity equation. The $\mathrm{QG}$ equations are more restrictive than the primitive equations, yet they are the starting point for much of terrestrial meteorology. The required assumptions are no less plausible for Jupiter than for the Earth, and certainly apply to many Jovian phenomena. We believe that these well-studied equations provide the best starting point for introducing multiple degrees of freedom in the vertical for models of Jupiter's atmosphere.

The advantages of the normal-mode model over the more common layer model are explained in detail by Flierl (1978). A major advantage of the former is that its derivation also produces a straightforward method for determining the model parameters given the vertical thermal structure of the atmosphere. For a layer model, on the other hand, the model parameters (thickness and density of the layers) are not uniquely determined for a given continuous vertical structure. Furthermore, a layer model does not accurately represent nonlinear interactions of baroclinic structures (Flierl 1978).

This paper is organized as follows. In section 2 we derive the equations for the normal-mode model, and discuss the lower boundary condition. Section 3 describes the vertical structure of Jupiter's atmosphere, and discusses the applicability of the normal-mode approach and the effect of the upper boundary condition. Sections 4 and 5 test our normal-mode model by solving simple problems using both the normal-mode model and a continuous vertical structure. These tests demonstrate that a normal-mode model is applicable to the study of large-scale Jovian atmospheric dynamics.

\section{Normal-mode model}

Our model is based on the quasi-geostrophic (QG) equation for conservation of potential vorticity on a $\beta$-plane in log-pressure coordinates (e.g., Gill 1982; Pedlosky 1987):

$$
\frac{D}{D t}\left(\nabla^{2} \psi+\beta y+\mathcal{L} \psi\right)=0
$$

where

$$
\begin{gathered}
\mathcal{L} a=e^{z} \frac{\partial}{\partial z}\left(\frac{e^{-z}}{L_{D}^{2}} \frac{\partial a}{\partial z}\right), \\
\frac{D a}{D t}=\frac{\partial a}{\partial t}+J(\psi, a), \\
J(a, b)=\frac{\partial a}{\partial x} \frac{\partial b}{\partial y}-\frac{\partial a}{\partial y} \frac{\partial b}{\partial x} .
\end{gathered}
$$

Here $\psi$ is the geostrophic streamfunction, $D / D t$ is the advective derivative, $z \equiv-\ln \left(p / p_{1}\right)$ with $p_{1}$ a reference pressure level, $L_{D}(z)=N H / f_{0}$ is the local internal deformation radius, $N$ is the Brunt-Väisälä frequency, $H$ $=R T / g$ is the pressure scale height, $T$ is the temperature, $R$ is the gas constant, $g$ is the gravitational acceleration, and $f=f_{0}+\beta y$ is the Coriolis parameter.

The important assumptions implicit in this QG formulation are: $H / L \ll 1$ where $H$ and $L$ are characteristic vertical and horizontal length scales respectively, $U / f L \ll 1$ where $U$ is a characteristic horizontal velocity, and $L / U \tau \ll 1$ where $\tau$ is a characteristic time for radiative and viscous dissipation. One further assumes that the frequencies are no larger than $U / L$ and that the scale $L$ is small compared to the planetary radius. The QG equations are usually derived under the assumption $L \sim L_{D}$, but the case $L_{D} \rightarrow 0$ can be handled with suitable upper and lower boundary conditions [see Eqs. (2.6) - (2.12) below].

Following Gavrilin (1965) and Flierl (1978), we separate out the vertical structure and write the streamfunction in the form

$$
\psi(x, y, z, t)=\sum_{n=0}^{N} \psi_{n}(x, y, t) \Phi_{n}(z),
$$

where the functions $\Phi_{n}(z)$ are solutions to the equation

$$
\mathcal{L} \Phi_{n}+\lambda_{n}{ }^{2} \Phi_{n}=0
$$

Boundary conditions are applied at $z_{0}$ and $z_{2}$. Equation (2.4) gives the vertical structure of a Rossby wave with deformation radius $\lambda_{n}{ }^{-1}$ (Pedlosky 1987). Substituting (2.3) and (2.4) into (2.1), multiplying by $e^{-\left(z-z_{1}\right)} \Phi_{n} d z$, and integrating from $z_{0}$ to $z_{2}$, we obtain a set of coupled equations for the $\psi_{n}$ :

$$
\begin{aligned}
\sum_{m=0}^{N} \delta_{m n} & {\left[\frac{\partial}{\partial t}\left(\nabla^{2} \psi_{m}-\lambda_{m}{ }^{2} \psi_{m}\right)+\beta \frac{\partial \psi_{m}}{\partial x}\right] } \\
& +\sum_{l, m=0}^{N} \gamma_{l m n} J\left(\psi_{l}, \nabla^{2} \psi_{m}-\lambda_{m}{ }^{2} \psi_{m}\right)=0,
\end{aligned}
$$


where

$$
\begin{gathered}
\delta_{m n}=\int_{z_{0}}^{z_{2}} e^{-\left(z-z_{1}\right)} \Phi_{m} \Phi_{n} d z, \\
\gamma_{l m n}=\int_{z_{0}}^{z_{2}} e^{-\left(z-z_{1}\right)} \Phi_{l} \Phi_{m} \Phi_{n} d z .
\end{gathered}
$$

We assume that the $\Phi_{n}$ are normalized so that $\delta_{n n}=1$, and we define a reference level $z_{1}$ in the range $z_{0}<z_{1}$ $<z_{2}$.

Application of this model is complicated by the lack of solid upper and lower boundaries. We focus on a thin, stably-stratified, weather layer $\left(z_{1}<z<z_{2}\right)$ that rests hydrostatically on a much thicker, neutrallystratified interior layer $\left(z_{0}<z<z_{1}\right)$. For the upper boundary condition $\left(z=z_{2}\right)$ we show in the next section that Jupiter's upper troposphere and stratosphere $(p \leqslant 700 \mathrm{mb}$ ) act almost as a rigid lid, reflecting $\sim 90$ percent of the energy propagating upward from below. Accordingly, we implement the normal-mode model with a rigid lid at $p=p_{2} \sim 700 \mathrm{mb}$. The bulk of the paper is devoted to testing the normal-mode and rigid lid assumptions by comparing solutions with these approximations to solutions of the continuous equations with an outgoing wave (radiation boundary condition) assumed at the top of the stratosphere $(p=1 \mathrm{mb})$.

For the lower boundary condition $\left(z=z_{0}\right)$, we assume that vertical motions are zero or suitably bounded at the base of the deep interior layer, whose thickness $\left(z_{1}-z_{0}\right)$ is much greater than the thickness of the upper weather layer $\left(z_{2}-z_{1}\right)$. As we will show, solving (2.4) under these assumptions yields a barotropic ( $n$ $=0)$ mode with $\lambda_{0}^{2}=0$ and $\Phi_{0}=$ constant in $z_{0}<z$ $<z_{2}$, plus a set of baroclinic $(n \geqslant 1)$ modes with $\lambda_{n}{ }^{2}$ $>0$ and nonzero amplitude $\Phi_{n} \neq 0$ only in the weather layer $z_{1}<z<z_{2}$. In other words, the appropriate boundary condition on the baroclinic modes at the base of the weather layer $\left(z=z_{1}\right)$ is $\Phi_{n}=0$.

This boundary condition for the baroclinic modes was discussed by Gierasch et al. (1979) and Conrath et al. (1981), although they did not consider a barotropic mode which extends into the deep adiabatic region below. Setting the barotropic mode to zero is equivalent to assuming solid-body rotation in Jupiter's interior up to the base of the water cloud (or other source of static stability). More general assumptions include steady, zonal flow $\psi_{0}=\psi_{0}(y)$, or the even more general time-dependent motions of a rotating, isentropic, spherical, fluid body (e.g., Busse 1976; Ingersoll and Pollard 1982; Ingersoll and Miller 1986).

Since the lower boundary condition for the baroclinic modes of the weather layer is crucial to what follows, we repeat the arguments of Gierasch et al. (1979) for the $\beta$-plane, focussing not on the dynamics of the lower region but rather on its effects at the base of the weather layer. To treat the case $L_{D} \rightarrow 0$, we decompose (2.1) into the QG vorticity equation and the thermodynamic energy equation:

$$
\begin{aligned}
\frac{D}{D t}\left(\nabla^{2} \psi+\beta y\right)-f_{0} e^{z} \frac{\partial}{\partial z}\left(e^{-z} w\right) & =0, \\
\frac{D}{D t}\left(\frac{\partial \psi}{\partial z}\right)+f_{0} L_{D}{ }^{2} w & =0 .
\end{aligned}
$$

Here $w=d z / d t$ is the vertical velocity in log-pressure coordinates. Consider a small-amplitude disturbance whose amplitude varies as $\exp (i k x+i l y+i \omega t)$. Equations (2.6) and (2.7) then become:

$$
\begin{array}{r}
i \omega \lambda^{2} \psi-f_{0} e^{z} \frac{\partial}{\partial z}\left(e^{-z} w\right)=0, \\
i \omega \frac{\partial \psi}{\partial z}+f_{0} L_{D}^{2} w=0, \\
\lambda^{2}=\left(\frac{k \beta}{\omega}\right)-\left(k^{2}+l^{2}\right) .
\end{array}
$$

These equations are equivalent to (2.4), but $\lambda^{2}$ is now a separation constant that depends upon the frequency and horizontal wavenumber.

For the lower region where $L_{D}{ }^{2}=0,(2.9)$ says that $\partial \psi / \partial z=0$. We exclude the possibility $\omega=0, \partial \psi / \partial z$ $\neq 0$ as it leads to infinitely small-scale oscillations $\left(\lambda^{2} \rightarrow \infty\right)$ in the upper weather layer according to (2.4) and (2.10). With $\psi=$ constant, (2.8) yields

$$
w=\frac{i \omega \lambda^{2} \psi}{f_{0}}\left[\exp \left(z-z_{0}\right)-1\right] \text { for } z_{0} \leqslant z \leqslant z_{1}
$$

where we have used the lower boundary condition $w$ $=0$ at $z=z_{0}$. At the base of the weather layer $\psi$ and $w$ are continuous, although $\partial \psi / \partial z$ and $L_{D}{ }^{2}$ may be discontinuous. Thus at $z=z_{1}$ we combine (2.9) and (2.11) to yield

$\left.\frac{\partial \psi}{\partial z}\right|_{z=z_{1}}+\left.\lambda^{2} L_{D}^{2}\left[\exp \left(z_{1}-z_{0}\right)-1\right] \psi\right|_{z=z_{1}}=0$

This is the required boundary condition at $z=z_{1}$ for small-amplitude QG motions in the weather layer. The barotropic mode satisfies (2.12) with $\lambda^{2}=0$ and $\partial \psi /$ $\partial z=0$. The lowest baroclinic modes have a small number of vertical oscillations in the upper weather layer and therefore have $\lambda^{2} L_{D}{ }^{2} \sim 1$. However, the great depth of the lower layer means that the exponential in (2.12) is large. This implies that the baroclinic modes have $\psi=0$ at $z=z_{1}$, as discussed earlier.

The above results lead to simplifications of Eq. (2.5). The baroclinic modes have $\Phi_{n}=0$ at $z=z_{1}$, and $\partial \Phi_{n} /$ $\partial z=0$ at $z=z_{2}$ which follows from (2.9) with a rigid lid $(w=0)$ at the upper boundary. Thus $(2.4)$ is a Sturm-Liouville system in $z_{1}<z<z_{2}$, with discrete eigenvalues $\lambda_{n}$ and orthogonal eigenfunctions $\Phi_{n}$ for $n$ $\geqslant 1$. The constant $\delta_{m n}$ is either 0 or 1 , depending upon whether $m \neq n$ or $m=n$, respectively. For the baro- 
clinic modes, the integrals in the definitions of $\delta_{m n}$ and $\gamma_{l m n}$ can be taken from $z_{1}$ to $z_{2}$. The magnitudes of the $\Phi_{n}$ will be of order unity if $z_{2}-z_{1}$ is of order unity. Setting the barotropic eigenfunction $\Phi_{0}$ equal to unity then ensures that all of the eigenfunctions are of the same magnitude in $z_{1}<z<z_{2}$.

The equation for the barotropic mode amplitude $\psi_{0}(x, y, t)$ is obtained from (2.5) by setting $n=0$. The integrals go from $z_{0}$ to $z_{2}$. With $\Phi_{0}=1$ we have $\delta_{00}$ $=\gamma_{000} \gg 1$. All the other constants $\delta_{m 0}$ and $\gamma_{l m 0}$ are of order unity and may be neglected. Since $\lambda_{0}^{2}=0$ we are left with

$$
\frac{\partial}{\partial t}\left(\nabla^{2} \psi_{0}\right)+\beta \frac{\partial \psi_{0}}{\partial x}+J\left(\psi_{0}, \nabla^{2} \psi_{0}\right)=0,
$$

which is the usual potential vorticity equation for a barotropic fluid with a rigid lid. Thus the barotropic mode is unaffected by the presence of baroclinic modes (or equivalently, the weather layer does not affect the interior), although the barotropic mode will affect the baroclinic modes.

The separation into a single barotropic mode and set of baroclinic modes with $\psi=0$ at $z \leqslant z_{1}$ does not require a rigid lower boundary at $z=z_{0}$. A scaling analysis of the nonlinear equation (2.6) gives the same result. The argument was given by Ingersoll and Cuong (1981) in the discussion of the $1^{1 / 2}$ layer model. If the magnitude of $w$ is everywhere consistent with QG scaling in the weather layer, then the great depth of the lower layer implies that the term involving $\partial / \partial z$ in (2.6) is negligible in the lower layer. This leaves

$$
\frac{\partial}{\partial t}\left(\nabla^{2} \psi\right)+\beta \frac{\partial \psi}{\partial x}+J\left(\psi, \nabla^{2} \psi\right)=0,
$$

which is the equation for the barotropic mode derived above. Thus the full equation in the interior region is satisfied by the barotropic mode alone. The baroclinic component of the solution is confined to the upper layer. Continuity of $\psi$ then requires $\Phi_{n}=0$ at $z=z_{1}$ for $n \geqslant 1$.

A final derivation of the lower boundary condition is given in section 4 of Gierasch et al. (1979). They include nonhydrostatic and ageostrophic effects in the lower layer. Equation (2.1) still holds in the upper layer, since frequencies are small compared to $f_{0}$, and $L$ $\sim L_{D}$. The motion in the lower layer is a downwardpropagating wave whose vertical wavelength is large compared to the horizontal wavelength. The vertical wavelength is therefore much larger than the upper layer thickness $\left(z_{2}-z_{1}\right)$. A parameter analogous to the ratio of lower layer thickness to upper layer thickness is large when the frequencies are small. The argument resulting in (2.12) is shown to hold for the nonhydrostatic case as well as for the hydrostatic case.

The derivations of the lower boundary condition by Gierasch et al. (1979) treat the adiabatic interior as an infinitely deep constant density fluid on a $\beta$-plane, ig- noring effects of compressibility and spherical geometry. Our derivation is quasi-geostrophic and ignores ageostrophic and nonhydrostatic effects. The general problem of how the neutrally stable fluid interior of a Jovian planet interacts with the stably stratified atmosphere is poorly understood, and further work is needed in this area.

The equations for the baroclinic mode amplitudes $\psi_{n}(x, y, t)$ are obtained from (2.5) with $n \geqslant 1$. A complication arises because the barotropic mode is not orthogonal to the baroclinic modes in $z_{1}<z<z_{2}$. Thus we have $\delta_{0 n}=\gamma_{00 n} \neq 0$. However, the terms that involve these constants cancel because of (2.13). In fact, the only terms involving $\psi_{0}$ in (2.5) are from the double sum with one of the summation indices equal to $n$ and the other equal to zero. This follows from $\Phi_{0}=1$, whence $\gamma_{0 m n}=\delta_{m n}$ which is zero unless $m=n$. With these special cases taken into account, the equation for the $n$th baroclinic mode is

$$
\begin{aligned}
& \frac{\partial}{\partial t}\left(\nabla^{2} \psi_{n}-\lambda_{n}{ }^{2} \psi_{n}\right)+\beta \frac{\partial \psi_{n}}{\partial x} \\
& +J\left(\psi_{0}, \nabla^{2} \psi_{n}-\lambda_{n}{ }^{2} \psi_{n}\right)+J\left(\psi_{n}, \nabla^{2} \psi_{0}\right) \\
& \quad+\sum_{l, m=1}^{N} \gamma_{l m n} J\left(\psi_{l}, \nabla^{2} \psi_{m}-\lambda_{m}{ }^{2} \psi_{m}\right)=0
\end{aligned}
$$

An energy equation can be obtained by multiplying (2.15) by $\psi_{n}$, integrating over $x$ and $y$, and summing over the modes $n$. Assuming periodic boundary conditions in $x$, and either periodicity in $y$ or $\partial \psi / \partial x=0$ and $\partial^{2} \bar{\psi} / \partial t \partial y=0$ at $y=y_{1}$ and $y=y_{2}$ (Holton 1979), where $\bar{\psi}$ is the $x$ average of $\psi$, we obtain

$$
\begin{aligned}
& \frac{\partial}{\partial t} \sum_{n} \iint \frac{1}{2}\left(\left|\nabla \psi_{n}\right|^{2}+\lambda_{n}^{2} \psi_{n}^{2}\right) d A \\
& =-\sum_{n} \iint \psi_{0} \frac{\partial}{\partial y}\left(\frac{\partial \psi_{n}}{\partial x} \nabla^{2} \psi_{n}\right) d A
\end{aligned}
$$

The integrand of the left hand side of (2.16) represents the total energy of mode $n$, while the right hand side describes the conversion of energy from the barotropic mode to the baroclinic modes. This term has the same form as the conversion from mean kinetic energy to eddy kinetic energy (Holton 1979), although our baroclinic modes can have a mean part. Also, our barotropic mode has such a large inertia that its amplitude does not change during the interaction.

As we are interested only in the behavior of the weather layer, we do not solve (2.14), but instead make a general assumption which guarantees that it is satisfied. Since $\psi_{0}=\psi_{0}(y)$ is an exact solution of (2.14), we assume that the motion in the deep interior, which corresponds to the barotropic mode, is steady and zonal (solid-body rotation is a special case of this). Although more complicated interior motions, which are not so- 
lutions of (2.14) but follow a more general equation for flow in a rotating sphere, are possible, we do not consider them in the context of this model. Regardless of the appropriate equation, a steady zonal flow is likely to be a possible solution for the motions in the interior.

Equation (2.15) may be truncated at any $N \geqslant 1$. The normal-mode model with $N=1$ is dynamically equivalent to the reduced gravity single layer model considered by Ingersoll and Cuong (1981). This equivalence may then be used to relate the single layer model to observations. To show this we set $N=1$ in (2.15) with $\psi_{0}=\psi_{0}(y)$. The equation for a single baroclinic mode then becomes

$$
\begin{array}{r}
\frac{\partial}{\partial t}\left(\nabla^{2} \psi_{1}-\lambda_{1}{ }^{2} \psi_{1}\right)+\beta \frac{\partial \psi_{1}}{\partial x}+J\left(\psi_{0}, \nabla^{2} \psi_{1}-\lambda_{1}{ }^{2} \psi_{1}\right) \\
+J\left(\psi_{1}, \nabla^{2} \psi_{0}+\gamma_{111} \nabla^{2} \psi_{1}\right)=0
\end{array}
$$

Defining $\hat{\psi}=\psi_{0}+\gamma_{111} \psi_{1}$ and $\lambda=\lambda_{1},(2.17)$ can be manipulated into the form

$$
\begin{aligned}
\frac{\partial}{\partial t}\left(\nabla^{2} \hat{\psi}-\lambda \hat{\psi}\right) & +\beta \frac{\partial \hat{\psi}}{\partial x} \\
& +J\left(\hat{\psi}, \nabla^{2} \hat{\psi}-\lambda^{2} \hat{\psi}+\lambda^{2} \psi_{0}\right)=0,
\end{aligned}
$$

which is the equation of motion for the single layer model of Ingersoll and Cuong (1981), where $\hat{\psi}$ is the streamfunction in the thin upper layer, $\psi_{0}$ is the streamfunction for the deep lower layer, and $\lambda^{-1}$ is the deformation radius for the upper layer. This correspondence allows us to relate the radius of deformation and velocity in the single layer model to observations. The observed streamfunction $\psi_{\text {obs }}$ is then related to the normal-mode model by

$$
\psi_{\mathrm{obs}}=\psi_{0} \Phi_{0}\left(z_{\mathrm{obs}}\right)+\psi_{1} \Phi_{1}\left(z_{\mathrm{obs}}\right),
$$

where $z_{\mathrm{obs}}$ is the level of the observations and $\Phi_{0}(z)$ $=1$. Substituting $\psi_{1}=\left(\hat{\psi}-\psi_{0}\right) / \gamma_{111}$ gives

$$
\psi_{\mathrm{obs}}=\frac{\Phi_{1}\left(z_{\mathrm{obs}}\right)}{\gamma_{111}} \hat{\psi}+\left(1-\frac{\Phi_{1}\left(z_{\mathrm{obs}}\right)}{\gamma_{111}}\right) \psi_{0} .
$$

In the next section, we find $\gamma_{111} \sim 1.17$ and $\Phi_{1}\left(z_{\text {obs }}\right)$ $\sim 1.22$ for our standard model of Jupiter's atmosphere described in the next section. This implies that the velocities for the upper layer of a single layer model, computed from $\hat{\psi}$, may be compared directly to velocities observed on Jupiter, with an error of around 5 percent. This simple correspondence between layer and normal-mode models only holds with one baroclinic mode, since in general a $N$-mode model has more free parameters than a $N$-layer model. (Flierl 1978 discusses the general problem of calibrating layer models.) The single layer model (2.18) conserves an energy-like quantity-the global integral of $\frac{1}{2}\left(|\nabla \hat{\psi}|^{2}+\lambda^{2} \hat{\psi}^{2}\right)$. This conservation law holds in addition to (2.16) with $N=1$, but it does not seem to extend to $N \geqslant 2$.

\section{Application of the model to Jupiter}

Knowledge of the vertical temperature structure allows us to apply this model to Jupiter. The crucial parameter is the Brunt-Väisälä frequency $N(z)$, which enters through the local deformation radius $L_{D}(z)$ $=N H / f_{0}$ in (2.2) and (2.4). The solutions of (2.4) determine the $\lambda_{n}$ and $\gamma_{l m n}$ according to (2.5), and these constants uniquely define the planet in the normalmode equation (2.15).

Figure 1 shows the temperature profiles measured during the Voyager radio occultation experiments (Lindal et al. 1981), which cover the pressure range from $1 \mathrm{mb}$ to $1000 \mathrm{mb}$, along with the temperature profile used in our calculations. We used the radio occultation profile for $p<690 \mathrm{mb}$, and a moist pseudoadiabat (discussed below) for $p>690 \mathrm{mb}$. The observed cloud-tracked winds refer to the range from approximately $500 \mathrm{mb}$ to $1000 \mathrm{mb}$. This is the location of the ammonia cloud, which is calculated to overlie deeper cloud layers of ammonium hydrosulfide and water (e.g., Weidenschilling and Lewis 1973). The base of the water cloud lies at 3-6 bars, depending upon the water abundance. Below this cloud, the temperature is thought to follow a dry adiabat, reflecting the fact that sunlight does not penetrate into the layers below, and the only source of energy is heat from Jupiter's interior.

Our fundamental assumption is that the observed winds are the surface manifestation of barotropic and baroclinic motions that extend from the ammonia cloud down to the base of the water cloud. This is the weather layer of the previous section. The static stability

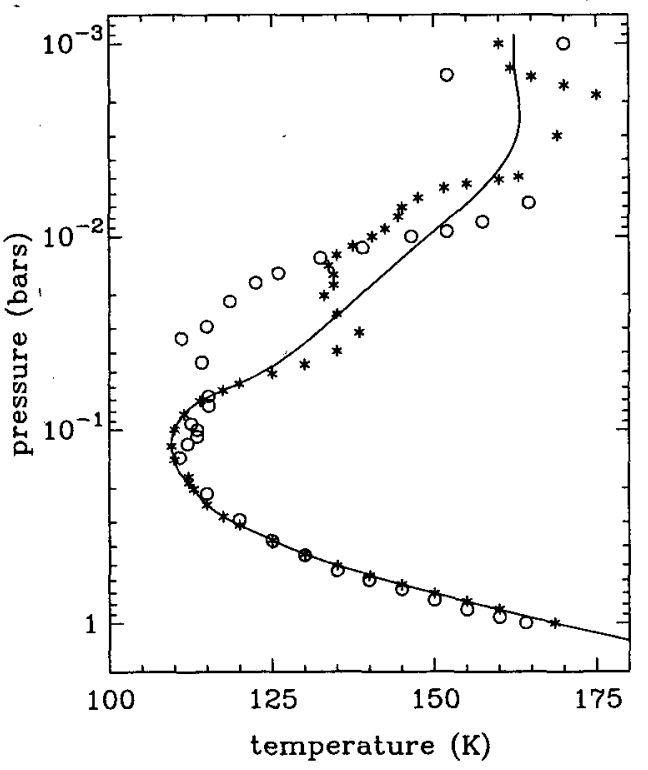

FIG. 1. Temperature profiles for Jupiter's atmosphere. The open circles are from the Voyager 2 radio occultation egress data, the asterisks are from the Voyager 2 radio occultation ingress data, and the solid line is the profile used in our models. 
of the layer, from which $N(z)$ and $L_{D}(z)$ are derived, is taken to be the difference between the assumed temperature profile and a dry adiabat. Justification for the last assumption comes from the earth's tropics, which are near neutral stability for moist convection, but whose large-scale motions are apparently governed by dry adiabatic processes (e.g., Holton 1979; Gill 1982). This assumption has been applied to Jupiter and Saturn in the past (e.g., Barcilon and Gierasch 1970; Allison and Stone 1983), but there are no direct observations to confirm or deny it.

The Brunt-Väisälä frequency is expressed in terms of the virtual temperature $T_{V}(T, p)$ as follows:

$$
\begin{aligned}
& N^{2}=\frac{g^{2}}{R_{d} T_{V}^{2}}\left(\frac{d T_{V}}{d z}+\frac{R_{d} T_{V}}{C_{p}(d)}\right), \\
& T_{V}=T /[1+(\epsilon-1) e(T) / p] .
\end{aligned}
$$

Here $R_{d}$ is the gas constant of the dry atmosphere, $\epsilon$ $=m_{v} / m_{d}$ is the ratio of the molecular weight of water vapor to that of the dry atmosphere, $e(T)$ is the saturation vapor pressure of the condensate, $C_{p}(d)$ is the specific heat of the dry atmosphere, and $z=-\ln \left(p / p_{1}\right)$ as before. The relation between $T$ and $z$ is taken to be a moist pseudoadiabat, in which moisture is removed as soon as it condenses. Thus we have (e.g., Weidenschilling and Lewis 1973)

$$
\frac{d T}{d z}=\frac{R T}{C_{\nu}} \frac{\left(1+\frac{L f_{m}}{R T}\right)}{\left(1+\frac{L^{2} \epsilon f_{m}}{R C_{p} T^{2}}\right)},
$$

where $R$ and $C_{p}$ are the gas constant and specific heat of the saturated mixture, respectively, $L$ is the latent heat per unit mass of the vapor, and $f_{m}$ is the mass fraction of water vapor in the saturated atmosphere. With these definitions, $N^{2}$ is the difference between $g(d \ln \rho / d h)$ for moist and dry adiabatic processes, respectively, where $h$ is the height in dimensional units. The idea is that the first term in (3.1) represents the temperature profile of the atmosphere, which is set by rapid convective motions occupying only a small fraction of the total surface area. This is analogous to cumulus convection in the Earth's tropics (Riehl and Malkus 1958; Palmén and Newton 1969). The presence of cumulus convection occupying a small fraction of the surface area is used by Lunine and Hunten (1987) to reconcile the observed low water abundance on Jupiter with the abundance predicted by models of planetary formation. The second term represents the collective behavior of the gas during relatively slow, large-scale motions.

The dry gas is assumed to be 89 percent hydrogen and 11 percent helium by volume. Our standard model has water vapor as the only condensable, owing to its relatively large abundance and dominant contribution to the latent heat. The base of the water cloud occurs where the saturation vapor pressure divided by the total pressure is equal to the subcloud mixing ratio. The cloud base is defined to be the bottom of the weather layer $\left(z=z_{1}\right)$ discussed in the preceding section. For comparison with the standard model, we added the effect of an ammonium hydrosulfide $\left(\mathrm{NH}_{4} \mathrm{SH}\right)$ cloud layer, which is predicted by chemical equilibrium calculations of the cloud structure (Lewis 1969; Weidenshilling and Lewis 1973), although the observational data are inconclusive (e.g., West et al. 1986). The $\mathrm{NH}_{4} \mathrm{SH}$ cloud produces a small spike in the $N^{2}$ profile between 1.3 and 2 bars, as shown in Fig. 2, but little change at other pressures. The probable ammonia cloud occurs at pressures less than $700 \mathrm{mb}$, where we are using the radio occultation data to determine the temperature structure.

Another problem in calculating the static stability of Jupiter's atmosphere is that the specific heat of hydrogen depends upon the ratio of ortho-hydrogen (parallel proton spin vectors) to para-hydrogen (antiparallel proton spins), and the rate at which equilibration between the two forms occurs (Massie and Hunten 1982; Conrath and Gierasch 1984). In our models, we assume that the ortho-hydrogen and para-hydrogen are in thermal equilibrium; this assumption is consistent with the Voyager IRIS data for Jupiter (Conrath and Gierasch 1984). The rate at which equilibration occurs is also important, as it determines if the heat released from conversion of ortho-hydrogen to para-hydrogen affects the temperature gradient. For frozen equilibrium hydrogen, the heat of conversion is ignored, while for equilibrium hydrogen it is included. The conditions

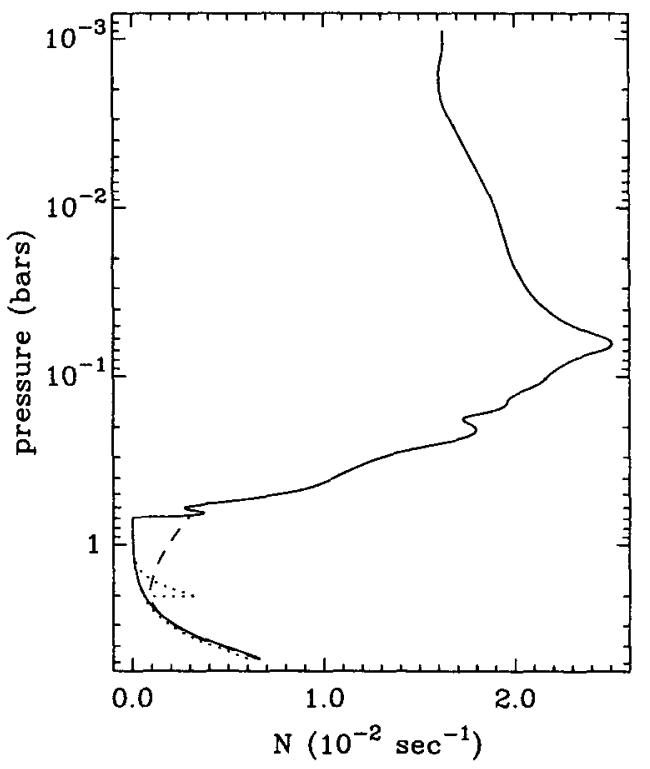

Fig. 2. Model static stability profile. The solid line is for the standard model. The dotted line is for the standard model with an $\mathrm{NH}_{4} \mathrm{SH}$ cloud. The dashed line is for equilibrium hydrogen. 
under which each of these assumptions holds are discussed in detail by Conrath and Gierasch (1984). One necessary condition for the equilibrium case to hold, that the convective time scale be at least as long as the equilibration time (one month or longer), seems at odds with the Voyager imaging sequences. Calculations of the heat of conversion are given by Massie and Hunten (1982). As shown in Fig. 2, the equilibrium case has a larger Brunt-Väisälä frequency than the frozen equilibrium case. For Jupiter, the difference in temperature gradient between the equilibrium and frozen equilibrium cases is smaller than the errors in measurement of the temperature gradient, so that either case is possible. On Uranus, however, the lower temperature produces a larger difference between the equilibrium and frozen equilibrium adiabats, and the observed temperature profiles are consistent only with frozen equilibrium (Gierasch and Conrath 1987). We therefore use frozen equilibrium in our standard model, although the equilibrium case is sometimes used for comparison. Since it has been suggested that the temperature gradient in Jupiter's lower troposphere is intermediate between the wet and dry adiabats, we also considered a model in which $N^{2}$ is one-half of the wet adiabatic value.

The final obstacle in applying the normal-mode model to Jupiter is the upper boundary condition. An ideal boundary condition would account for all possible processes occurring above the top of the modeled region, and is not feasible. If we assume that there are no mechanical energy sources or partially reflecting layers above the top of the model so that all energy leaving out the top escapes, and that any interactions between modes above the top of the modeled region are negligible, we may use the radiation condition: there is no downward directed energy flux at the top of the model. The radiation condition is still unwieldy with our normal-mode model since the radiation condition will not in general allow normal modes with real eigenvalues $\lambda_{n}$ and orthogonal eigenfunctions. $\Phi_{n}$. To obtain real eigenvalues and orthogonal eigenfunctions, we assume the rigid-lid boundary condition $d \Phi_{n} / d z$ $=0$ (Pedlosky 1987). A rigid lid, however, has the side effect of reflecting energy incident upon it (Lindzen et al. 1967). But if the atmosphere without the lid has a level at or below which a large amount of upwardly propagating energy is reflected back down, the region below this level will behave essentially like an atmosphere with a lid, and the use of an artificial lid is justified. We may thus test the rigid lid approximation by. calculating how well the model atmosphere with the outgoing wave upper boundary condition traps vertically propagating energy. This is done in several ways. The first is by calculating how well upward propagating waves are reflected back downward by the upper atmosphere as measured by a reflection coefficient. The second is by determining if there are almost-resonant modes at discrete $\lambda_{n}$. Other tests, involving forced
Rossby waves and baroclinic instability are discussed in the next two sections.

To implement the radiation condition, we assume constant temperature above $1 \mathrm{mb}$ (where the Voyager radio occultation data ends), and note that the general solution to (2.4) in this isothermal region is then

$$
\Phi_{n}=A \exp \left[\left(\frac{1}{2}+i q\right) z\right]+B \exp \left[\left(\frac{1}{2}-i q\right) z\right]
$$

where $A$ and $B$ are complex constants and

$$
q=\left(\lambda_{n}^{2} L_{D}^{2}-\frac{1}{4}\right)^{1 / 2}
$$

with $L_{D}$ a constant. If we calculate the energy flux for this solution, we find that the first term corresponds to an upward energy flux and the second term to a downward energy flux, so that we need $B=0$. Taking the ratio of $\Phi_{n}$ to $\partial \Phi_{n} / \partial z$, we obtain the upper boundary condition for a purely outgoing wave:

$$
\frac{\partial \Phi_{n}}{\partial z}=\left(\frac{1}{2}+i q\right) \Phi_{n}
$$

Calculation of reflection coefficients is done by a method similar to that used by Halevy and Peltier (1985) for barotropic waves. We assume a purely outgoing wave at $1 \mathrm{mb}$, and determine the ratio of the downward to upward wave amplitude at $z=z_{1}$. This ratio is defined as the reflection coefficient $\mathscr{R}$. Since $\mathscr{R}$ is the ratio of wave amplitudes, the fraction of energy which is reflected is given by $\mathscr{R}^{2}$. To calculate $\mathscr{R}$, we integrate (2.4) down from $1 \mathrm{mb}$, with (3.5) as the upper boundary condition, to the base of the water cloud using a fourth order Runge-Kutta method. An artificial region with constant $L_{D}=L_{D}\left(z_{1}\right)$ is added below the base of the water cloud. In this region, (2.4) has (3.4) as an analytic solution. Since $L_{D}$ is continuous, the boundary of this region with the water cloud is nonreflecting, so that $\mathscr{R}$ is independent of the details of the added lower region. Applying (3.4) and its first derivative in the added region provides a set of two equations in the two unknowns $A$ and $B$, which can be solved for $\mathcal{R}=|B| A \mid$. A similar calculation was done assuming $L_{D}=L_{D}\left(z_{1}\right) \exp \left(z_{1}-z\right)$ in the added region. This functional form also has an analytic solution that allows the upward and downward propagating waves to be identified. As expected, the assumed form for $L_{D}$ did not affect the results.

We use a similar method to determine the presence of almost-resonant modes. Equation (2.4) is integrated from the top of the model using boundary condition (3.5), to the bottom of the weather layer, where we evaluate a response function

$$
r(\lambda)=\left(\frac{1}{L_{D}^{2}}\left|\frac{\partial \Phi}{\partial z}\right|_{z=z_{1}}^{2}+\lambda^{2}|\Phi|_{z=z_{1}}^{2}\right) / \lambda^{2}|\Phi|_{z=z_{1}}^{2}
$$


The numerator is related to the energy density evaluated at the lower boundary. The denominator is the square of the forcing amplitude, chosen to make $r(\lambda)$ $\geqslant 1$. Without forcing the denominator would be zero, since $\Phi\left(z_{1}\right)=0$ when the conditions of the preceding section are satisfied. We define an almost resonant mode as a local maxima of $r(\lambda)$ with $r \gg 1$. Although the choice of response function is somewhat arbitrary (Lindzen and Tung 1976), we tried several response functions, all of which gave similar results.

The results of these calculations are shown in Fig. 3 for the standard model at $30^{\circ}$ latitude [note that $\lambda_{i}$ $\propto f_{0} \propto \sin$ (latitude) ] for a water mixing ratio of $10^{-3}$ by volume. The reflection coefficient $R$ varies from 0.90 to 0.95 , with small scale variations due to details of the static stability in the stratosphere, and the response function shows strong peaks at discrete $\lambda_{n}$ corresponding to deformation radii $\lambda_{n}{ }^{-1}$ of 735,155 , and $88 \mathrm{~km}$. A look at the $\Phi_{n}$ corresponding to the response peaks, as seen in Fig. 4, shows that each one has $n$ -1 zeroes in the pressure range between $700 \mathrm{mb}$ and the cloud base-a property of eigenfunctions with a rigid lid. The large reflection coefficients and sharp response peaks are due entirely to vertical variations of the static stability, in contrast to the Earth's atmosphere where variations of the vertical wind shear are necessary to achieve large reflection coefficients (Holton 1979).

Figure 5 shows the reflection coefficient and response function with the $\mathrm{NH}_{4} \mathrm{SH}$ cloud added. The reflection coefficient is reduced somewhat for values of $\lambda^{-1}$ between approximately 100 and $500 \mathrm{~km}$. The amplitude of the response peaks are reduced over the standard

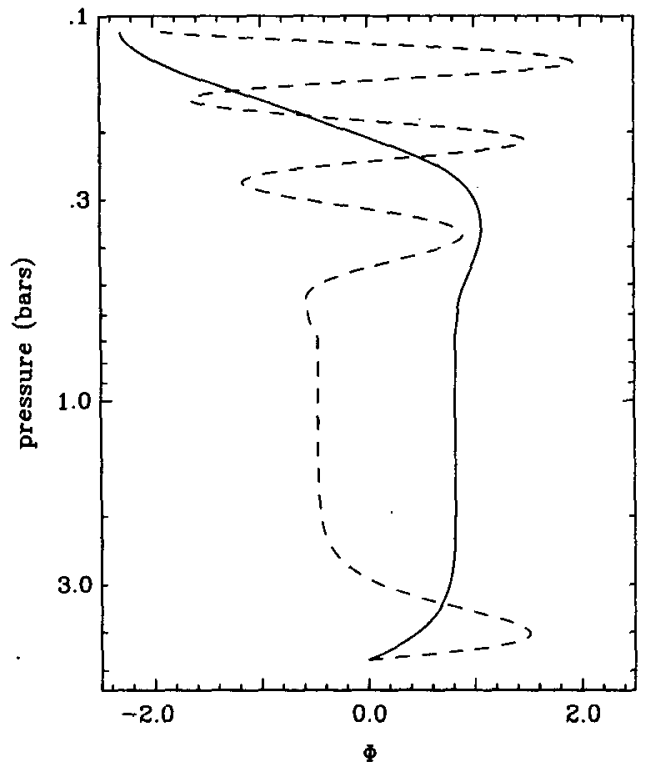

Fig. 4. Vertical structure $\Phi_{i}$ for the first two peaks in the response function in the standard model, shown at the phase when $\partial \Phi_{i} /\left.\partial z\right|_{z=z_{1}}$ is a maximum. The solid line is for $\lambda^{-1}=735 \mathrm{~km}$. The dashed line is for $\lambda^{-1}=155 \mathrm{~km}$.

model, but are still relatively large. Figure 6 shows the same calculations repeated using equilibrium hydrogen and no $\mathrm{NH}_{4} \mathrm{SH}$ : the reflection coefficient decreases strongly for $\lambda>3 \times 10^{-3} \mathrm{~km}^{-1}$. While the response peaks occur at similar $\lambda$ as the standard model, only the first peak is strong and sharp. The other peaks are

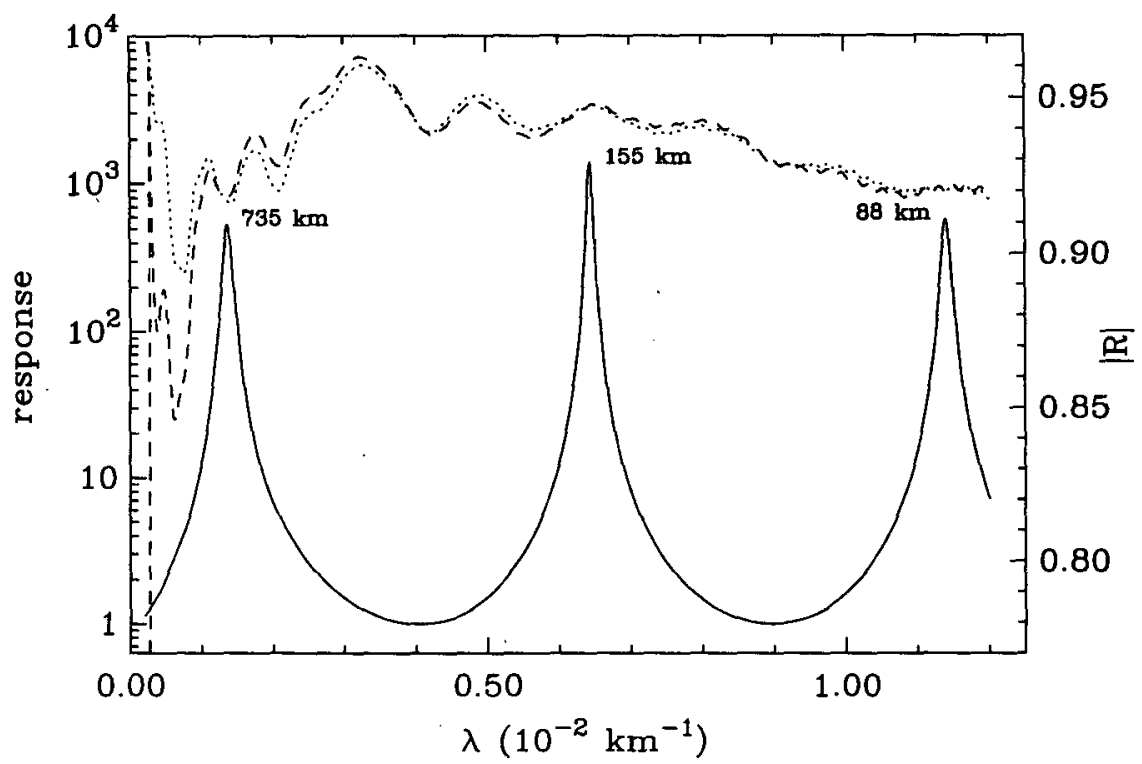

FIG. 3. Reflection coefficients and response function for the standard model. The solid line is the response function. The dashed line is the reflection coefficient with $L_{D}\left(z<z_{1}\right)=L_{D}\left(z_{1}\right)$. The dotted line is the reflection coefficient with $L_{D}\left(z<z_{1}\right)=L_{D}\left(z_{1}\right) \exp \left(z_{1}-z\right)$. Numeric labels are the deformation radii $\left(\lambda^{-1}\right)$ corresponding to peaks in the response function. 


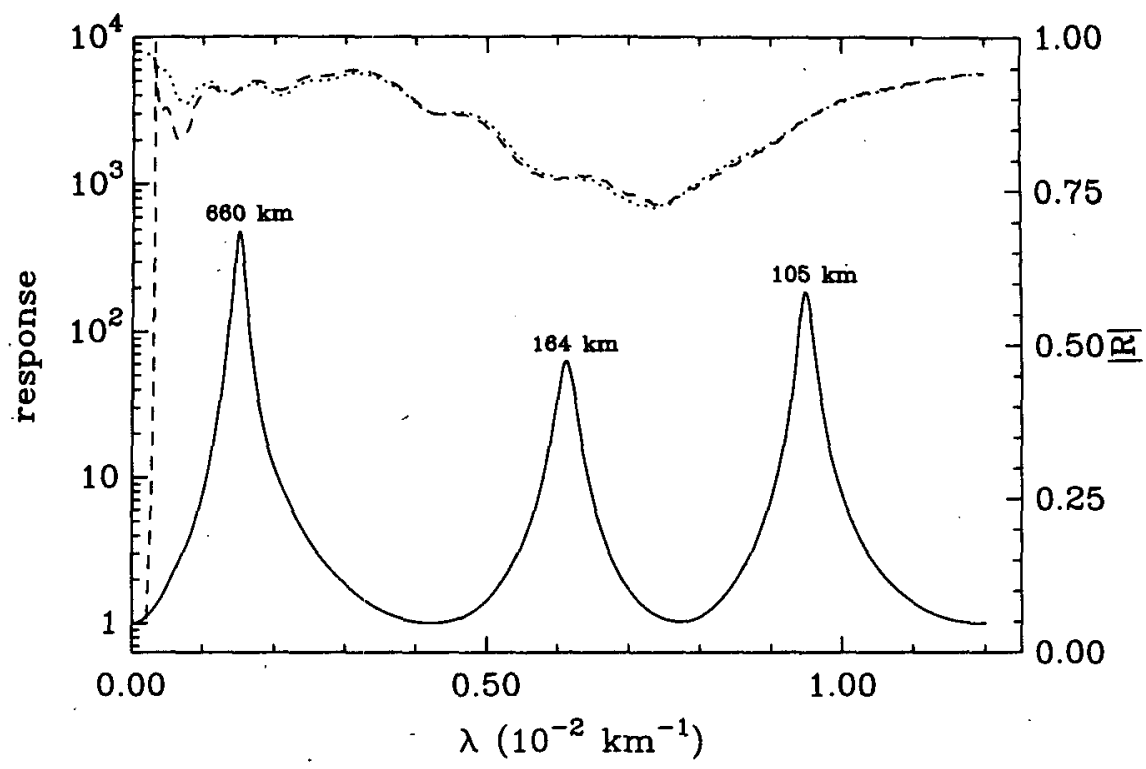

Fig. 5. Reflection coefficients and response function for the standard model with an added $\mathrm{NH}_{4} \mathrm{SH}$ cloud. The solid line is the response function. The dashed line is the reflection coefficient with $L_{D}\left(z<z_{1}\right)=L_{D}\left(z_{1}\right)$. The dotted line is the reflection coefficient with $L_{D}\left(z<z_{1}\right)$ $=L_{D}\left(z_{1}\right) \exp \left(z_{1}-z\right)$. Numeric labels are the deformation radii $\left(\lambda^{-1}\right)$ corresponding to peaks in the response function.

low and broad, as would be expected with a low reflection coefficient. As discussed earlier, the equilibrium hydrogen model does not appear to work for Uranus, and we do not expect it to apply to Jupiter. We also did these calculations for a value of $N^{2}$ equal to one- half the wet adiabatic value. This reduction of $N^{2}$ has very little effect on either the reflection coefficient or the amplitude of the response peaks, although the values of $\lambda$ at the peaks are larger by a factor of approximately $\sqrt{2}$. The variation in results for the different

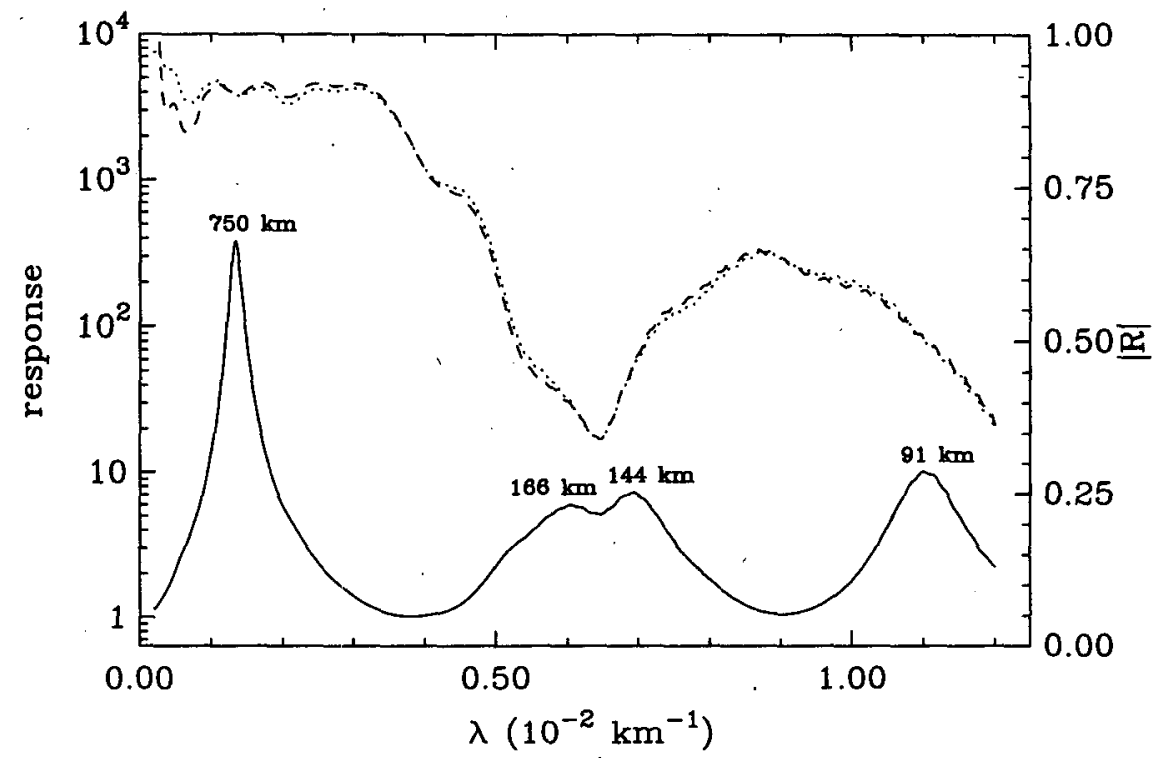

FIG. 6. Reflection coefficients and response function for equilibrium hydrogen. The solid line is the response function. The dashed line is the reflection coefficient with $L_{D}\left(z<z_{1}\right)=L_{D}\left(z_{1}\right)$. The dotted line is the reflection coefficient with $L_{D}\left(z<z_{1}\right)=L_{D}\left(z_{1}\right) \exp \left(z_{1}-z\right)$. Numeric labels are the deformation radii $\left(\lambda^{-1}\right)$ corresponding to peaks in the response function. 
assumptions indicates that the response function provides a sensitive test for determining the applicability of the rigid lid and normal-mode approximations.

Since our standard model has a high reflection coefficient and sharp resonances, we are allowed to use a rigid lid. We put this lid at $690 \mathrm{mb}$. This choice makes the $n$th mode of the model with a lid correspond roughly to the $n$th response peak of the model without a lid for $n=1,2$, and 3 . With the lid in place, we can calculate the eigenvalues and eigenfunctions of (2.4), and the interaction coefficients $\gamma_{l m n}$. The eigenfunctions are normalized so that $\delta_{n n}=1$. The structure of the modes is nearly identical to the functions shown in Fig. 4 for $p>690 \mathrm{mb}$ except for the normalization: the normalized modes have $\Phi_{1}(690 \mathrm{mb})=1.26$, and $\Phi_{2}(690 \mathrm{mb})=-0.708$. The values of $\lambda_{n}{ }^{-1}$ and $\gamma_{l m n}$ are summarized in Table 1 for various values of the water abundance (note that $f_{\mathrm{H}_{2} \mathrm{O}}$ in Table 1 is the mole fraction). The deformation radii $\lambda_{n}{ }^{-1}$ vary approximately as the square root of the water abundance. Such variation is expected from (3.1) and (3.3), which indicate that $N$ is proportional to $f_{\mathrm{H}_{2} \mathrm{O}}^{1 / 2}$, whence $L_{D}$ and $\lambda_{n}{ }^{-1}$ should also vary as $f_{\mathrm{H}_{2} \mathrm{O}}^{1 / 2}$ according to $(2.2)$ and (2.4). Although we do not show figures, the reflection coefficients remain high and the response functions remain peaked in the range examined $\left(10^{-4} \leqslant f_{\mathrm{H}_{2} \mathrm{O}} \leqslant 5\right.$ $\left.\times 10^{-3}\right)$. The values for $f_{\mathrm{H}_{2} \mathrm{O}}=10^{-3}$ are used for all subsequent calculations involving the normal-mode model with a lid.

\section{Test of normal-mode model: Forced Rossby waves}

A further test of the normal-mode approach is to apply broadband forcing at the base of the water cloud $\left(z=z_{1}\right)$ using the radiation condition (3.5) at $1 \mathrm{mb}$. We then determine how well that solution is approximated in $z_{1}<z<z_{2}$ by an expansion in the eigenfunctions obtained with a lid. By "broadband" we mean that the amplitude imposed at $z=z_{1}$ is independent of $\lambda$, which is related to the frequency and horizontal wavenumber by $(2.10)$. We expect the spectral density $d$ (energy) $/ d \lambda$ to look something like the response curve in Fig. 3, with most of the energy near discrete values of $\lambda$. To the extent that the vertical structure of the forced solutions at these frequencies

TABLE 1. Parameters of Jupiter normal-mode models.

\begin{tabular}{crrrrrr}
\hline \hline$f_{\mathrm{H}_{2} \mathrm{O}}$ & $\lambda_{1}{ }^{-1}$ & $\lambda_{2}{ }^{-1}$ & $\gamma_{111}$ & $\gamma_{112}$ & $\gamma_{122}$ & $\gamma_{222}$ \\
\hline $5 \times 10^{-3}$ & 1550 & 389 & 1.184 & -0.1757 & 0.7936 & 1.182 \\
$2 \times 10^{-3}$ & 985 & 228 & 1.174 & -0.1574 & 0.8029 & 1.304 \\
$1 \times 10^{-3}$ & 693 & 154 & 1.176 & -0.1506 & 0.8125 & 1.368 \\
$5 \times 10^{-4}$ & 486 & 106 & 1.184 & -0.1470 & 0.8228 & 1.418 \\
$2 \times 10^{-4}$ & 300 & 64 & 1.200 & -0.1453 & 0.8375 & 1.470 \\
$1 \times 10^{-4}$ & 208 & 44 & 1.214 & -0.1454 & 0.8497 & 1.503 \\
\hline
\end{tabular}

Note: the units of $\lambda_{n}^{-1}$ are kilometers and are for $\pm 30^{\circ}$ latitude; $\lambda_{l m n}$ are dimensionless; $f_{\mathrm{H}_{2} \mathrm{O}}$ is the water mole fraction. resembles the eigenfunctions obtained with a lid at 690 $\mathrm{mb}$, the normal mode approximation is good.

We represent the forced wave as a sum over $\lambda$ :

$$
\psi(x, y, z, t)=\sum_{\lambda}\left(\hat{\phi}_{r} \cos \alpha+\hat{\phi}_{i} \sin \alpha\right),
$$

where $\hat{\phi}(\lambda ; z)$ are complex solutions of $(2.4)$ subject to the radiation condition (3.5), and $\hat{\phi}_{r}$ and $\hat{\phi}_{i}$ are the real and imaginary parts of $\hat{\phi}$, respectively. The $\dot{\phi}$ are normalized so that $|\hat{\phi}|=1$ at $z=z_{1}$, corresponding to a forcing amplitude independent of $\lambda$. The sum in (4.1) includes numerous values of $\lambda$ on and off the peaks in the response curve. At each $\lambda$ the phase $\alpha$ of the forcing is arbitrary; we have

$$
\alpha(\lambda ; x, y, t)=k x+l y+\omega t+\alpha^{\prime}(\lambda),
$$

where $\alpha^{\prime}(\lambda)$ is a random number between 0 and $2 \pi$, independent of the $\alpha^{\prime}$ at all other values of $\lambda$.

Specifying all the $\alpha$ 's constitutes a single realization of the possible vertical profiles of $\psi$. Within $z_{1}<z$ $<z_{2}$, this vertical profile can be represented as an expansion in the real eigenfunctions $\Phi_{n}(z)$ obtained with the rigid lid at $z_{2}$. The coefficients $b_{n}$ of this expansion are obtained by minimizing the integrated variance, defined as

$\sigma_{N}^{2}=\left\langle\left[\sum_{\lambda}\left(\hat{\phi}_{r} \cos \alpha+\hat{\phi}_{i} \sin \alpha\right)-\sum_{n=1}^{N} b_{n} \Phi_{n}\right]^{2}\right\rangle$

where

$$
\langle X\rangle=\int_{z_{1}}^{z_{2}} X e^{-\left(z-z_{1}\right)} d z .
$$

Differentiating with respect to $b_{n}$ and using the orthonormal property of the $\Phi_{n}$, namely $\left\langle\Phi_{m} \Phi_{n}\right\rangle=\delta_{m n}$, we obtain

$$
b_{n}=\sum_{\lambda}\left(\left\langle\hat{\phi}_{r} \Phi_{n}\right\rangle \cos \alpha+\left\langle\hat{\phi}_{i} \Phi_{n}\right\rangle \sin \alpha\right) .
$$

Substituting (4.5) into (4.3) gives

$$
\begin{aligned}
\sigma_{N}^{2}=\left\langle\left\{\sum _ { \lambda } \left[\left(\hat{\phi}_{r}-\sum_{n=1}^{N}\left\langle\hat{\phi}_{r} \Phi_{n}\right\rangle \Phi_{n}\right) \cos \alpha\right.\right.\right. \\
\left.\left.\left.+\left(\hat{\phi}_{i}-\sum_{n=1}^{N}\left\langle\hat{\phi}_{i} \Phi_{n}\right\rangle \dot{\Phi}_{n}\right) \sin \alpha\right]\right\}^{2}\right\rangle .
\end{aligned}
$$

We average over all possible phases $\alpha(\lambda)$ of the continuous solution to obtain an average measure of the error $\bar{\sigma}_{N}^{2}$ in using the normal-mode solution. Using the fact that the $\alpha$ are independent and the $\Phi_{n}$ are orthonormal, we find

$$
\begin{aligned}
\bar{\sigma}_{N}{ }^{2}=\frac{1}{2} \sum_{\lambda}\left[\left\langle\hat{\phi}_{r}{ }^{2}\right\rangle\right. & +\left\langle\hat{\phi}_{i}{ }^{2}\right\rangle \\
& \left.-\sum_{n=1}^{N}\left(\left\langle\hat{\phi}_{r} \Phi_{n}\right\rangle^{2}+\left\langle\hat{\phi}_{i} \Phi_{n}\right\rangle^{2}\right)\right]
\end{aligned}
$$


A dimensionless measure of error is obtained by comparing this best fit variance $\bar{\sigma}_{N}^{2}$ to the variance $\bar{\sigma}_{0}^{2}$ with no fit at all. Calling the ratio of these $\chi^{2}$, we define

$$
\chi_{N}^{2}=\frac{\bar{\sigma}_{N}^{2}}{\bar{\sigma}_{0}^{2}},
$$

where

$$
\bar{\sigma}_{0}^{2}=\frac{1}{2} \sum_{\lambda}\left[\left\langle\hat{\phi}_{r}^{2}\right\rangle+\left\langle\hat{\phi}_{i}^{2}\right\rangle\right]
$$

Calculations were performed using the standard model, with $N=2$ and $N=3$, corresponding to 2 mode and 3-mode expansions, respectively. Since $\lambda^{2}$ can be either positive or negative according to (2.10), summations over $\lambda$ were done using both real and imaginary $\lambda$. When $\lambda$ is imaginary, the outgoing wave condition (3.5) is replaced by the requirement that $\Phi$ decays exponentially with altitude. The negative- $\lambda^{2}$ solutions are non-resonant (response functions are of order unity), and therefore these solutions do not contribute appreciably to the magnitudes of $\psi$ or $\chi_{N}{ }^{2}$. We summed over $|\lambda| \leqslant \lambda_{\max }$, using two values of $\lambda_{\max }$, namely $9.0 \times 10^{-3} \mathrm{~km}^{-1}$ and $1.4 \times 10^{-2} \mathrm{~km}^{-1}$, with equally spaced intervals in $\lambda$ of $10^{-5} \mathrm{~km}^{-1}$. The values of $\lambda_{\max }$ were chosen near the minimum of the response function following the $N=2$ and $N=3$ response peaks of the standard case. The resulting normalized errors are $\chi_{2}{ }^{2}=0.0152$ for $\lambda_{\max }=9 \times 10^{-3} \mathrm{~km}$, and $\chi_{2}{ }^{2}$ $=0.296$ and $x_{3}{ }^{2}=0.0116$ for $\lambda_{\max }=1.4 \times 10^{-2} \mathrm{~km}$. These numbers indicate that the normal-mode expansion provides a good approximation to the actual structure of uniformly forced waves, provided all of the modes with $\lambda_{n}<\lambda_{\max }$ are included. For the equilibrium hydrogen case, for which the reflection coefficients are not close to one (see Fig. 6), the values of $\chi_{2}{ }^{2}, \chi_{3}{ }^{2}$, and $\chi_{4}{ }^{2}$ are $0.211,0.144$, and 0.0472 , respectively, when $\lambda_{\max }=1.4 \times 10^{-2}$.

\section{Test of normal-mode model: Baroclinic instability}

As a final test of the normal-mode model, we consider the problem of pure baroclinic instability. Solutions to this problem for three different models are compared: a continuous model with a radiation condition at $1 \mathrm{mb}$, a continuous model with a rigid lid at $690 \mathrm{mb}$, and our standard normal-mode model with $N=2$. This allows us to determine the effects both of a rigid lid and of the normal-mode approximation separately. In all cases, we use the standard model of the thermal structure, with solid body rotation in the interior (i.e., $\psi_{0}=0$ ). We assume a basic state velocity given by

$$
\bar{u}(z)=U_{1} \Phi_{1}(z)+U_{2} \Phi_{2}(z),
$$

where $\Phi_{1}$ and $\Phi_{2}$ are the first two baroclinic eigenfunctions of the normal-mode expansion and the $U_{n}$ are given constants. The basic state streamfunction is then

$$
\bar{\psi}(y, z)=-U_{1} y \Phi_{1}(z)-U_{2} y \Phi_{2}(z) .
$$

To determine the baroclinic stability of the basic state, we add an infinitesimal perturbation of the form $\psi^{\prime}(z)$ $\times \exp (i k x-i k c t)$ to the basic flow and solve for the phase speed $c$ as a function of $U_{1}, U_{2}$ and $k$. If $c$ has an imaginary component, the perturbation will grow exponentially with growth rate $k c_{i}$ until nonlinear effects become important, and the basic flow is linearly unstable.

To solve this problem using the $N=2$ normal-mode model, we expand $\psi^{\prime}$ in our orthonormal eigenfunctions $\Phi_{n}$ :

$$
\psi^{\prime}(z)=\psi_{1}^{\prime} \Phi_{1}(z)+\psi_{2}^{\prime} \Phi_{2}(z) .
$$

Substituting (5.2) and (5.3) into (2.15), we obtain a set of equations for $\psi_{n}^{\prime}$ :

$$
\begin{aligned}
& \left(A_{11}+c\right) \psi_{1}^{\prime}+A_{12} \psi_{2}^{\prime}=0, \\
& A_{21} \psi_{1}^{\prime}+\left(A_{22}+c\right) \psi_{2}^{\prime}=0,
\end{aligned}
$$

where

$$
\begin{aligned}
& A_{11}=\left(\beta-k^{2}\left(\gamma_{111} U_{1}+\gamma_{112} U_{2}\right)\right. \\
& \left.-\gamma_{112}\left(\lambda_{1}^{2}-\lambda_{2}^{2}\right) U_{2}\right) /\left(k^{2}+\lambda_{1}^{2}\right), \\
& A_{12}=\left(-k^{2}\left(\gamma_{112} U_{1}+\gamma_{122} U_{2}\right)\right. \\
& \left.+\gamma_{112}\left(\lambda_{1}^{2}-\lambda_{2}^{2}\right) U_{1}\right) /\left(k^{2}+\lambda_{1}^{2}\right), \\
& A_{21}=\left(-k^{2}\left(\gamma_{112} U_{1}+\gamma_{122} U_{2}\right)\right. \\
& \left.-\gamma_{122}\left(\lambda_{1}{ }^{2}-\lambda_{2}{ }^{2}\right) U_{2}\right) /\left(k^{2}+\lambda_{2}{ }^{2}\right), \\
& A_{22}=\left(\beta-k^{2}\left(\gamma_{122} U_{1}+\gamma_{222} U_{2}\right)\right. \\
& \left.+\gamma_{122}\left(\lambda_{1}^{2}-\lambda_{2}^{2}\right) U_{1}\right) /\left(k^{2}+\lambda_{2}^{2}\right) \text {. }
\end{aligned}
$$

This can be treated as'a matrix eigenvalue problem, and will have nontrivial solutions only when $\operatorname{det} \mid \mathbf{A}$ $+c||=0$, which can be solved for the phase speed $c$ to obtain

$c=-\frac{1}{2}\left(A_{11}+A_{22}\right) \pm\left[\frac{1}{4}\left(A_{11}-A_{22}\right)^{2}+A_{12} A_{21}\right]^{1 / 2}$.

If the quantity in square brackets is negative, the phase speed of the perturbation is complex and the flow is linearly unstable. For known values of $\beta, \gamma_{l m n}$ and $\lambda_{n}{ }^{2}$, the 2-mode instability problem has three independent parameters: $k^{2}$, which determines the length scale of the perturbation, $s=U_{2} / U_{1}$, which determines the shape of the unperturbed basic state velocity profile, and $U=\operatorname{sgn}\left(U_{1}\right)\left(U_{1}^{2}+U_{2}^{2}\right)^{1 / 2}=U_{1}\left(1+s^{2}\right)^{1 / 2}$, which determines the amplitude of the basic state. It is then straightforward to calculate $c\left(s, U, k^{2}\right)$ from (5.6) and map out the regions of stable and unstable flow. 
Calculations were performed with the standard model with $f_{\mathrm{H}_{2} \mathrm{O}}=10^{-3}$. Calculations were done first with $s=1.769$, which is a jet with $\bar{u}=0$ at $z=z_{1}$ and $z=z_{2}$, and second with $s=-0.214$, which is a shear profile with $d \bar{u} / d z=0$ at $z=z_{1}$ and $z=z_{2}$. These velocity profiles are shown in Fig. 7. Contour plots of the growth rates are shown in Fig. 8 for $s=1.769$ and in Fig. 9 for $s=-0.214$. Velocities of order $1 \mathrm{~m} \mathrm{~s}^{-1}$ are required for the flow to become unstable. Figures 10 and 11 show growth rate contours in the $k^{2}-s$ plane for velocities more typical for Jupiter $(U=79.5$ $\mathrm{m} \mathrm{s}^{-1}$ ). At these large $U, s<0$ is more unstable than $s>0$, and for $s<0$ only a small amount of the second mode is needed for instability, although $s=0$ is stable. For large values of $U$, the $\beta$ terms in (5.5) are negligible for all growing disturbances. The remaining terms are proportional to $U$, so the phase speed and growth rate become proportional to $U$ for large $U$. It can be shown that $s=0$ (a pure first mode) is always stable in the 2 -mode model. This is done by noting that $c$ can have an imaginary part only if $A_{12} A_{21}<0$. However, with $U_{2}=s=0, A_{12} A_{21}$ is proportional to $\left(k^{2}-\lambda_{1}{ }^{2}+\lambda_{2}{ }^{2}\right)$ with a positive constant of proportionality. In this case, $A_{12} A_{21}>0$, since $\lambda_{1}{ }^{2}<\lambda_{2}{ }^{2}$. The shape of the profiles for various positive and negative $s$ have the same general properties as the cases described above.

We now wish to compare these results for the 2mode model to the models with continuous vertical structure. With a basic state velocity $\bar{u}(z)$ as given in (5.1) and perturbations of the form $\psi^{\prime}(z) \exp (i k x$ $-i k c t)$, the equation for the vertical structure of the disturbance is (Pedlosky 1987)

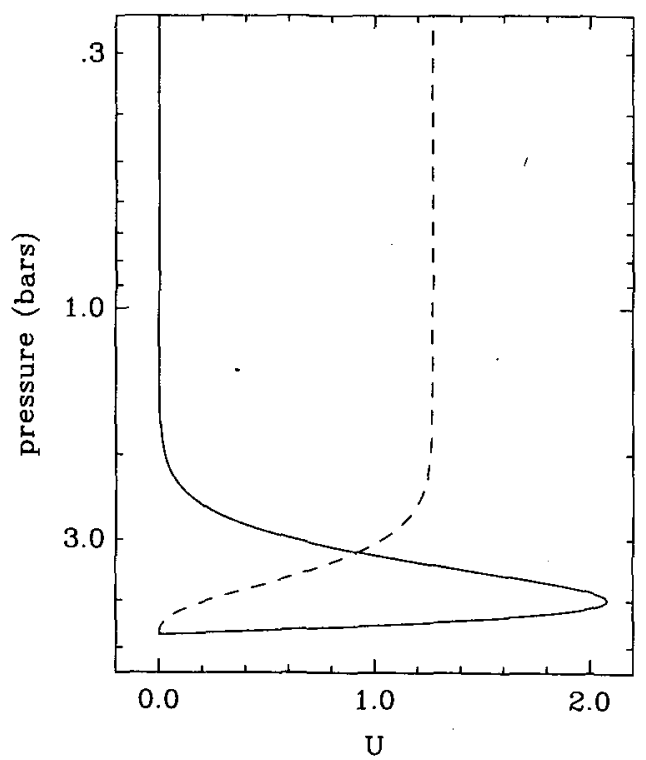

FIG. 7. Velocity profiles used in baroclinic instability calculations for $s=1.769$ (solid line) and $s=-0.214$ (dashed line) with $U=1$ $\mathrm{m} \mathrm{s}^{-1}$. At pressures greater than $690 \mathrm{mb}$, these profiles are essentially linear combinations of the curves shown in Fig. 4.

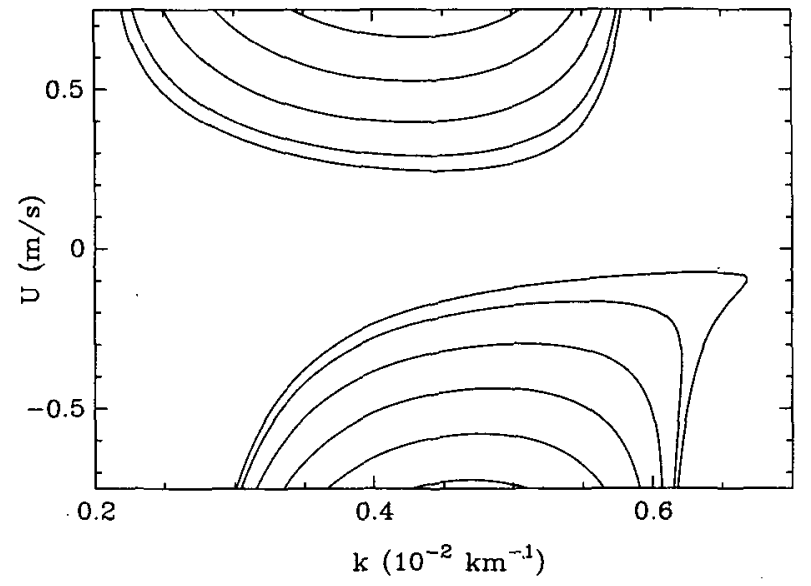

FIG. 8. Contour plot of growth rates for $s=1.769$. The contour interval is $2 \times 10^{-7} \mathrm{~s}^{-1}$ and the outer contour is the zero contour.

$$
(\bar{u}-c)\left(\mathcal{L} \psi^{\prime}-k^{2} \psi^{\prime}\right)+(\beta-\mathcal{L} \bar{u}) \psi^{\prime}=0
$$

The lower boundary condition is $\psi^{\prime}\left(z_{1}\right)=0$, as derived in section 2. In solving (5.7), we consider two upper boundary conditions: a rigid lid at $690 \mathrm{mb}$ (the same boundary condition used in the 2-mode model), and a radiation condition imposed at $1 \mathrm{mb}$. For the second case, $\bar{u}(z)$ is assumed constant above $690 \mathrm{mb}$ so that instability will only occur in the region covered by the normal-mode model. More general basic states which do not have $\partial \bar{u} / \partial z=0$ for $p \leqslant 690 \mathrm{mb}$ are possible, but are not considered in this paper. This problem can again be treated as an eigenvalue problem for the phase speed $c$.

The problem is solved by integrating (5.7) from the upper to lower boundary by a fourth order RungeKutta scheme and defining a complex function $F(c)$ as the value of $\psi^{\prime}\left(z_{1}\right)$ (i.e., the error in satisfying the lower boundary condition). The eigenvalues then correspond to the zeroes of $F(c)$, which are found by Newton-Raphson iteration, using the results of the 2-

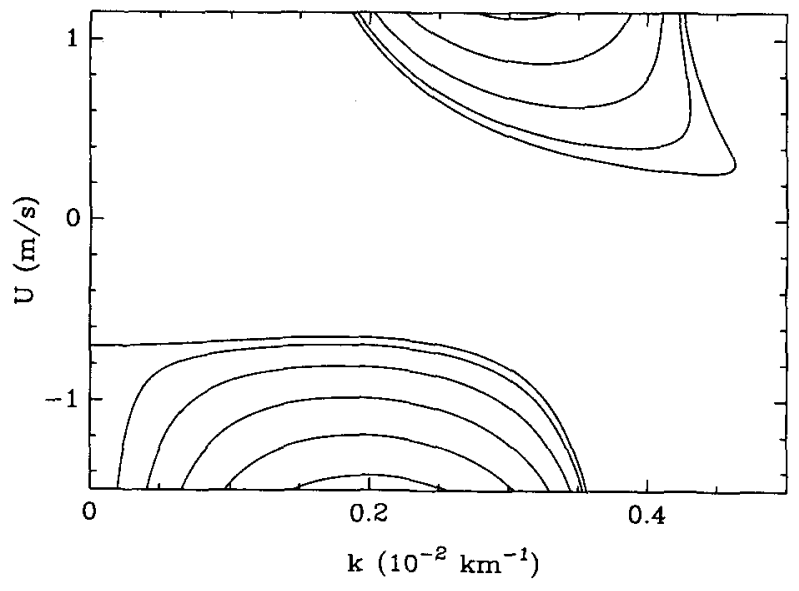

FIG. 9. As in Fig. 8 but for $s=-0.214$. 


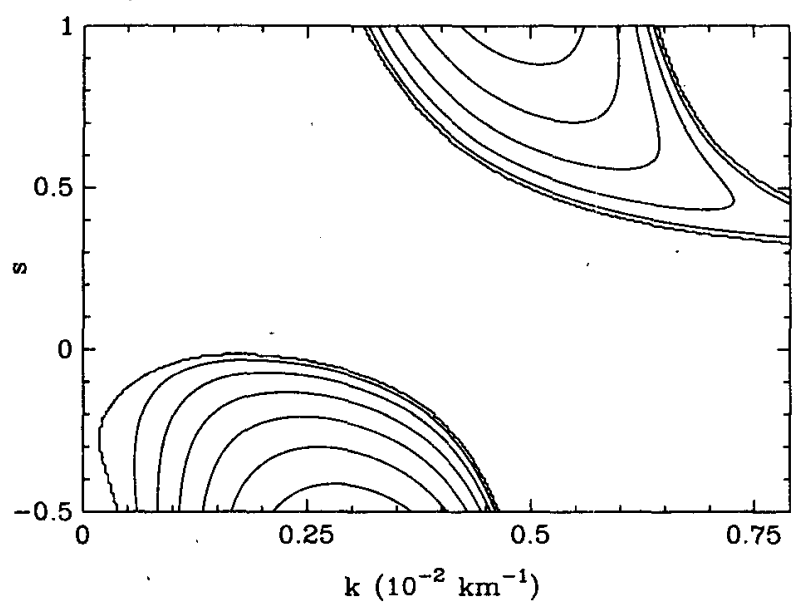

FiG. 10. Contour plot of growth rates for $U=79.5 \mathrm{~m} \mathrm{~s}^{-1}$. The contour interval is $5 \times 10^{-6} \mathrm{~s}^{-1}$ and the outer contour is the zero contour.

mode model as an initial guess. To avoid problems in integrating through critical levels (where $\vec{u}=c$ ), we only solved the continuous problem in the region of instability, where $c$ has an imaginary component. Also, we used different resolutions to make sure that the step size did not affect the results.

Typical results are presented in Figs. 12-14, which show vertical and horizontal cuts through the curves of Figs. 8 and 10. Figure 12 shows a comparison of the phase speeds and growth rates for continuous and 2mode solutions with $s=1.769, U=0.531 \mathrm{~m} \mathrm{~s}^{-1}$ and variable $k$. This case is somewhat unusual in that the 2-mode model has growth rates slightly lower than both of the continuous models, with and without the lid; the normal-mode model generally tends to overestimate growth rates. The growth rates of the normalmode model also drop off more sharply at the edge of the unstable region than both continuous models. This effect was observed in all of our calculations. The calculations also show that the effect of the rigid-lid ap-

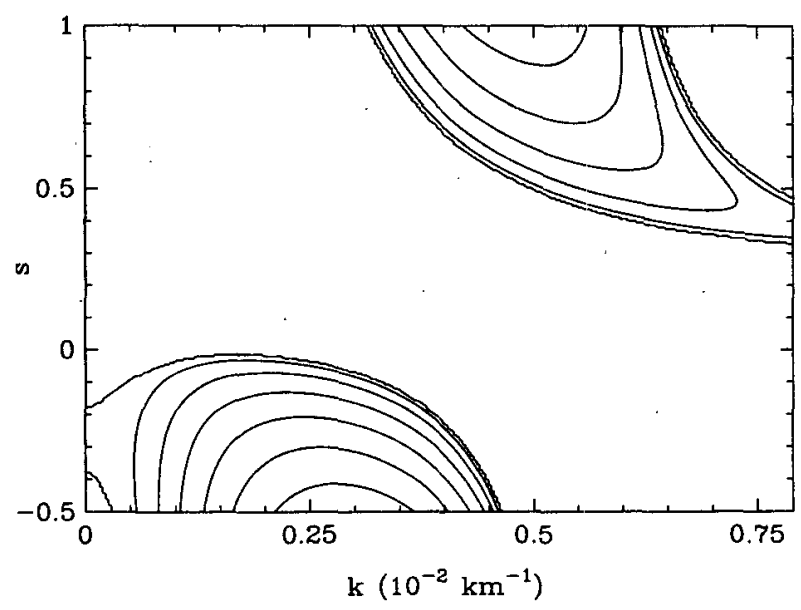

FIG. 11. As in Fig. 10 but for $U=-79.5 \mathrm{~m} \mathrm{~s}^{-1}$.

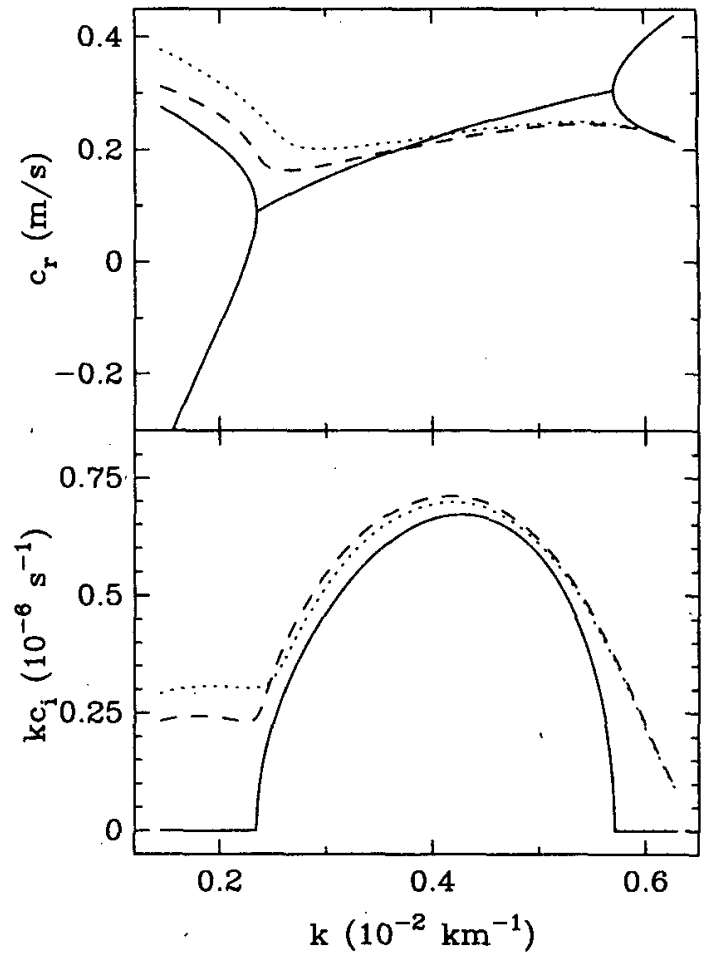

FIG. 12. Phase speed and growth rates for $s=1.769, U=0.531$ $\mathrm{m} \mathrm{s}^{-1}$, representing a horizontal cut through the upper lobe of Fig. 8. The solid line is the 2-mode solution, the dashed line is the continuous solution with a rigid lid, and the dotted line is the continuous solution with the radiation condition.

proximation decreases with increasing wavenumber. Despite the differences at the edge of the unstable region, the normal-mode solution approximates the continuous solutions fairly well around the most unstable wavelength. Figure 13 shows a comparison between the 2-mode and continuous solutions for $s$ $=1.769, k=4.56 \times 10^{-3} \mathrm{~km}^{-1}$ and $U$ varying. The normal-mode solution approximates the continuous solution quite well, slightly overestimating the growth rates, except at the boundary of the unstable region. Figure 14 compares 2-mode and continuous solutions for $k=1.69 \times 10^{-3} \mathrm{~km}^{-1}, U=-79.5 \mathrm{~m} \mathrm{~s}^{-1}$ and variable $s$. For this case, the 2 -mode model again overestimates the growth rates, and the effect of the upper boundary condition on the solution is fairly large due to the small wavenumber. In general, although the normal-mode model usually overestimates growth rates and underestimates the size of the unstable regions, it provides a good enough approximation around the most unstable wavelengths to be usable in calculations where a larger number of vertical degrees of freedom is impractical.

\section{Conclusions}

In this paper, we have proposed a normal-mode model for the dynamics of Jupiter's atmosphere, in 


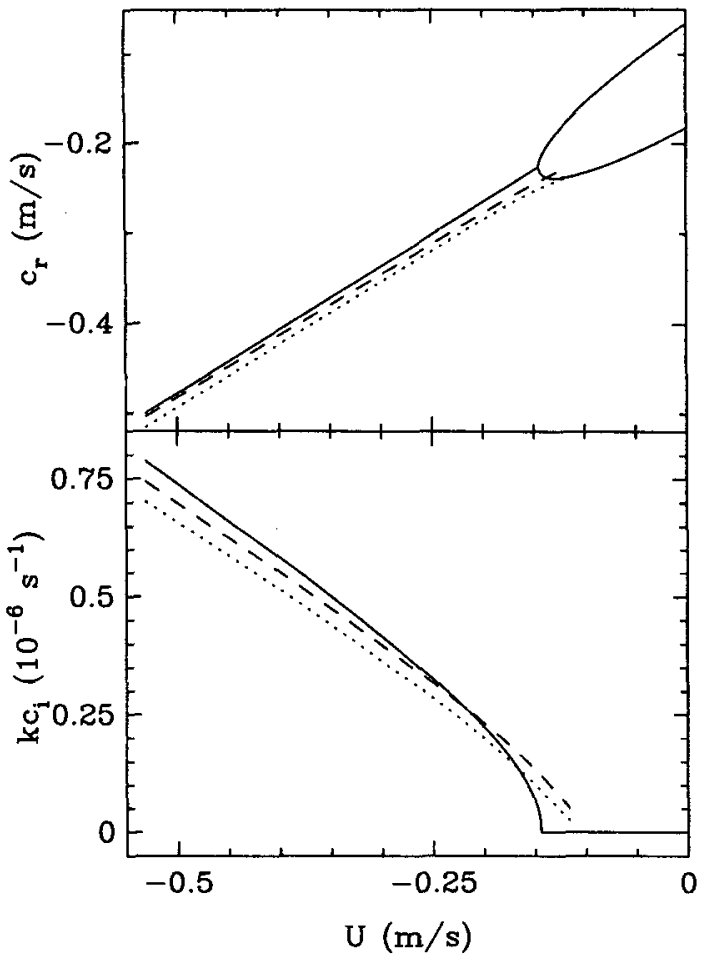

FiG. 13. As in Fig. 12 but for $s=1.769, k=4.56 \times 10^{-3} \mathrm{~km}^{-1}$ representing a vertical cut through the lower lobe of Fig. 8.

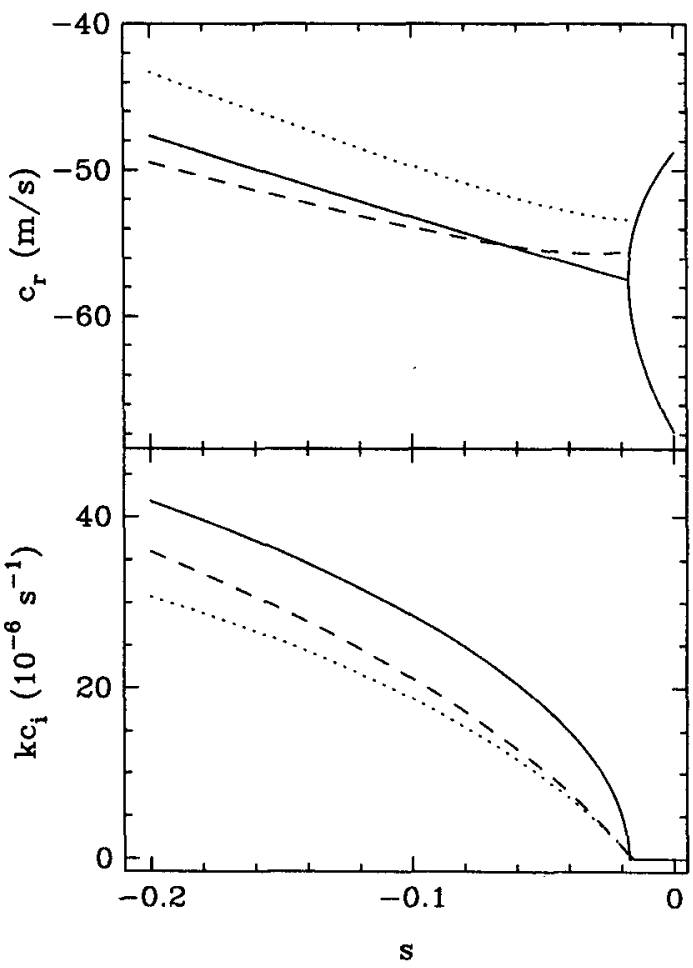

FIG. 14. As in Fig. 12 but for $k=1.69 \times 10^{-3} \mathrm{~km}^{-1}, U=-79.5$ $\mathrm{m} \mathrm{s}^{-1}$, representing a vertical cut through the lower lobe of Fig. 10. which the vertical structure is represented by a summation over orthogonal functions which are solutions to the equation for the vertical structure of Rossby waves. In section 2 we developed the relevant equations for a Jovian planet with a deep, adiabatic, fluid interior. One consequence of the adiabatic interior is that the model with one baroclinic mode is dynamically equivalent to the one-layer model commonly used to study Jovian atmospheric dynamics. Thus, calculations like those in section 3 can also be used to calibrate one layer models. For example, using our standard case (frozen equilibrium hydrogen with a volume mixing ratio of water of $10^{-3}$ ), we find that the proper deformation radius is $\sim 735 \mathrm{~km}$ for a latitude of \pm 30 degrees, and that velocities in the layer model can be approximately compared directly to the observations [see Eq. (2.20)] .

Calculation of reflection coefficients shows that our standard model has a stratosphere that reflects most ( $\sim 90$ percent) of the energy incident upon it. This leads to nearly resonant modes at discrete values of the deformation radius $\lambda^{-1}$, making the rigid-lid approximation viable and allowing us to calculate a set of orthonormal eigenfunctions for use in the normalmode expansion. Results in which $\mathrm{NH}_{4} \mathrm{SH}$ contributes to $N_{2}$ are similar to the standard model, although the reflection coefficients are somewhat lower. Results with equilibrium hydrogen are not as good. The reflection coefficient is only near one when $\lambda \leqslant 3 \times 10^{-3} \mathrm{~km}^{-1}$ so that only one near-resonant mode occurs. In this case the usefulness of the normal-mode is restricted to a single baroclinic mode. The same calculations can be done for any given model of the thermal structure to determine the applicability of a normal-mode expansion.

We further tested the normal-mode model on the problems of forced Rossby waves and baroclinic instability. In section 4 we demonstrated that the response to broadband forcing is sufficiently concentrated near the resonances for the result to be well approximated by the eigenfunction expansion. We then compared a two-mode model of pure baroclinic instability to the continuous problem. The results show that the twomode model provides a reasonably good approximation in the wavelengths around the maximum growth rate, so that the normal-mode model provides a simple, qualitatively correct method of including baroclinic effects into models of Jupiter's atmospheric dynamics.

Acknowledgments. This research was supported by the NASA Planetary Atmospheres program under Grant NAGW-58.

\section{REFERENCES}

Allison, M., and P. H. Stone, 1983: Saturn meteorology: A diagnostic assessment of thin-layer configurations for the zonal flow. Icarus, 54, 296-308.

Barcilon, A., and P. Gierasch, 1970: A moist Hadley cell model for Jupiter's cloud bands. J. Atmos. Sci., 27, 550-560. 
Bjoraker, G. L., H. P. Larson and V. G. Kunde, 1986a: The abundance and distribution of water vapor in Jupiter's atmosphere. Astrophys. J., 311, 1058-1072.

$\longrightarrow,-$ and $-1986 \mathrm{~b}$ : The gas composition of Jupiter derived from 5- $\mu \mathrm{m}$ airborne spectroscopic observations. Icarus, 66, 579609.

Busse, F. H., 1976: A simple model of convection in the Jovian atmosphere. Icarus, 29, 255-260.

Conrath, B. J., and P. J. Gierasch, 1984: Global variation of the para hydrogen fraction in Jupiter's atmosphere and implications for dynamics on the outer planets. Icarus, 57, 184-204.

- , and - 1986: Retrieval of ammonia abundances and cloud opacities on Jupiter from Voyager IRIS spectra. Icarus, 67, 444455.

- - and N. Nath, 1981: Stability of zonal flows on Jupiter. Icarus, 48, 256-282.

Dowling, T. E., and A. P. Ingersoll, 1988: Potential vorticity and layer thickness variations in the flow around Jupiter's Great Red Spot and White Oval BC. J. Atmos. Sci., 45, 1380-1396.

$\longrightarrow$, and - 1989: Jupiter's Great Red Spot as a shallow water system. J. Atmos. Sci., in press

Flierl, G. R., 1978: Models of vertical structure and the calibration of two-layer models. Dyn. Atmos. Oceans, 2, 341-381.

Gavrilin, B. L., 1965: On the description of vertical structure of synoptical processes. Izv. Atmos. Ocean. Phys., 1, 8-17.

Gierasch, P. J., and B. J. Conrath, 1987: Vertical temperature gradients on Uranus: Implications for layered convection. $J$. Geophys. Res., 92, 15 019-15 029.

-, and J. A. Magalhães, 1986: Zonal mean properties of Jupiter's upper troposphere from Voyager infrared observations. Icarus, 67, 456-483.

- A. P. Ingersell and D. Pollard, 1979: Baroclinic instabilities in Jupiter's zonal flow. Icarus, 40, 205-212.

Gill, A. E., 1982: Atmosphere-Ocean Dynamics. Academic Press.

Halevy, I., and W. R. Peltier, 1985: Barotropic instability and Rossby wave radiation. J. Atmos. Sci., 42, 1825-1837.

Hatzes, A., D. D. Wenkert, A. P. Ingersoll and G. E. Danielson, 1981: Oscillations and velocity structure of a long-lived cyclonic spot. J. Geophys. Res., 86, 8745-8749.

Holton, J. R., 1979: An Introduction to Dynamic Meteorology, second edition. Academic Press.

Ingersoll, A. P., and P. G. Cuong, 1981: Numerical model of longlived Jovian vortices. J. Atmos. Sci., 38, 2067-2076.

- and D. Pollard, 1982: Motion in the interiors and atmospheres of Jupiter and Saturn: scale analysis, anelastic equations, barotropic stability criterion. Icarus, 52, 62-80.

- and R. L. Miller, 1986: Motions in the interiors and atmospheres of Jupiter and Saturn 2. Barotropic instabilities and normal modes of an adiabatic planet. Icarus, 65, 370-382.

- R. F. Beebe, J. L. Mitchell, G. W. Garneaul, G. M. Yagi and J.-P. Müller, 1981: Interaction of eddies and mean zonal flow on Jupiter as inferred from Voyager 1 and 2 inages. J. Geophys. Rés., 86, 8733-8743.

,,-- B. J. Conrath and G. E. Hunt, 1984: Structure and dynamics of Saturn's atmosphere. Saturn, T. Gehrels and M. S. Matthews, Eds., University of Arizona Press, Tucson, 195-238.

Lewis, J. S., 1969: The clouds of Jupiter and the $\mathrm{NH}_{3}-\mathrm{H}_{2} \mathrm{O}$ and $\mathrm{NH}_{3}-$ $\mathrm{H}_{2} \mathrm{~S}$ systems. Icarus, 10, 365-378.
Limaye, S. S., 1986: Jupiter: new estimates of the mean zonal flow at the cloud level. Icarus, 65, 335-352.

- H. H. Revercomb, L. A. Sromovsky, R. J. Krauss, D. Santek, V. E. Suomi, S. A. Collins and C. C. Avis, 1982: Jovian winds from Voyager 2: Part I. Zonal mean circulation. J. Atmos. Sci., 39, 1413-1432.

Lindal, G. F., G. E. Wood, G. S. Levy, J. D. Anderson, D. N. Sweetnam, H. B. Hotz, B. J. Buckles, D. P. Holmes, P. E. Doms, V. R. Eshelman, G. L. Tyler and T. A. Croft, 1981: The atmosphere of Jupiter: An analysis of the Voyager radio occultation measurements. J. Geophys. Res., 86, 8721-8727.

Lindzen, R. S., and K.-K. Tung, 1976: Banded convective activity and ducted gravity waves. Mon. Wea. Rev., 104, 1602-1617.

- E. S. Batten and J. W. Kim, 1967: Oscillations in atmospheres with tops. Mon. Wea. Rev., 95, 441-451.

Lunine, J. I., and D. M. Hunten, 1987: Moist convection and the abundance of water in the troposphere of Jupiter. Icarus, 69 , $566-570$.

Mac Low, M.-M., and A. P. Ingersoll, 1986: Merging of vortices in the atmosphere of Jupiter: an analysis of Voyager images. Icarus, 65, 353-369.

Marcus, P. S., 1988: A numerical simulation of the Great Red Spot of Jupiter. Nature, 331, 693-696.

Massie, S. T., and D. M. Hunten, 1982: Conversion of para and ortho hydrogen in the Jovian planets. Icarus, 49, 213-226.

Maxworthy, T., and L. G. Redekopp, 1976: A solitary wave theory of the Great Red Spot and other observed features in the Jovian atmosphere. Icarus, 29, 261-271.

Mitchell, J. L., R. F. Beebe, A. P. Ingersoll and G. W. Garneau, 1981: Flow fields within Jupiter's Great Red Spot and White Oval BC. J. Geophys. Res., 86, 8751-8757.

Palmén. E., and C. W. Newton, 1969: Atmospheric Circulation Systems. Academic Press.

Pedlosky, J., 1987: Geophysical Fluid Dynamics, second ed. SpringerVerlag.

Pollack, J. B., M. Podolak, P. Bodenheimer and B. Christofferson, 1986: Planetesimal dissolution in the envelopes of the forming, giant planets. Icarus, 67, 409-443.

Read, P. L., 1986: Stable, baroclinic eddies on Jupiter and Saturn: A laboratory analog and some observational tests. Icaris, 65, 304-334.

__, and R. Hide, 1984: An isolated baroclinic eddy as a laboratory analogue of the Great Red Spot on Jupiter. Nature, 308, 4548.

Riehl, H., and J. S. Malkus, 1958: On the heat balance in the equatorial trough zone. Geophysica, 6, 503-537.

Weidenschilling, S. J., and J. S. Lewis, 1973: Atmospheric and cloud structures of the Jovian planets. Icarus, 20, 465-476.

West, R. A., D. F. Strobel and M. G. Tomasko, 1986: Clouds, -aerosols and photochemistry in the Jovian atmosphere. Icarus, 65, 161217.

Williams, G. P., 1975: Jupiter's atmospheric circulation. Nature, 257, 778 .

__, 1979: Planetary Circulations: 2 . The Jovian quasi-geostrophic regime. J. Atmos. Sci., 36, 932-968.

solitary vortices and Jovian eddies. J. Atmos. Sci., 41, 453-478.

, and R. J. Wilson, 1988: The stability and genesis of Rossby vortices, J. Atmos. Sci., 45, 207-241. 